Revue d'histoire de l'enfance « irrégulière »

Le Temps de l'histoire

4 | 2002

Images de l'enfance et de la jeunesse "irrégulières »

\title{
Le studio Henri Manuel et le ministère de la Justice : une commande non élucidée
}

Françoise Denoyelle

\section{(2) OpenEdition \\ Journals}

Édition électronique

URL : http://journals.openedition.org/rhei/56

DOI : $10.4000 /$ rhei.56

ISBN : 978-2-7535-1642-7

ISSN : $1777-540 \mathrm{X}$

Éditeur

Presses universitaires de Rennes

Édition imprimée

Date de publication : 15 novembre 2002

Pagination : 127-143

ISSN : 1287-2431

Référence électronique

Françoise Denoyelle, « Le studio Henri Manuel et le ministère de la Justice : une commande non élucidée », Revue d'histoire de l'enfance « irrégulière » [En ligne], 4| 2002, mis en ligne le 18 mai 2007, consulté le 03 décembre 2020. URL : http://journals.openedition.org/rhei/56 ; DOI : https://doi.org/ 10.4000/rhei.56 


\section{Le studio Henri Manuel et le ministère de la Justice : une commande non élucidée}

Bien avant la campagne contre les bagnes d'enfants lancée en août 1934 après l'évasion de colons de la maison d'éducation surveillée de Belle-Îleen-Mer, entre 1929 et $1931,{ }^{(2)}$ le studio Henri Manuel réalise une commande sur les prisons et les institutions pour mineurs relevant du ministère de la Justice. Le reportage est d'importance et couvre l'École d'administration pénitentiaire, 17 maisons d'arrêt, 6 centrales et enfin 9 établissements pour mineurs : les maisons d'éducation surveillée d'Aniane, de Belle-Île-en-Mer, de Saint-Maurice, la colonie correctionnelle d'Eysses, l'école de réforme de Saint-Hilaire, les écoles de préservation de Cadillac, de Clermont-de-l'Oise, de Doullens, ainsi que la prison de la Petite Roquette qui, à cette époque, accueille des mineurs. Pour certains établissements, le reportage donne lieu à deux albums, un pour les hommes et un pour les femmes. Quelle est l'origine de la commande, quel en est le commanditaire, à quel public s'adresse-t-elle ? Les questions sont nombreuses, les réponses ne peuvent guère dépasser le stade des hypothèses de travail. Comme pour de nombreux fonds photographiques, nous ne possédons que des images. Elles sont montées sous forme d'albums de facture artisanale. Les photographies sont collées sur du papier Canson et rassemblées par établissement. Seule la dénomination officielle de l'institution, inscrite à la main, figure sur la couverture, mais aucune légende n'accompagne les clichés. À ce jour, nous n'avons retrouvé aucune archive concernant les modalités et les finalités de cette commande. Restent plus d'une trentaine d'albums et des images extraites des reportages sur les institutions accueillant des mineurs et diffusées sous forme de carnets d'une douzaine de cartes postales.

\section{Françoise \\ Denoyelle $^{(1)}$}

(1) Maître de

conférences à l'ENS

Louis Lumière, Noisy-le-

Grand (93).

(2) Les dates inscrites sur les tableaux dans les classes ou affichées sur les éphémérides dans les bureaux permettent de dater précisément le reportage.

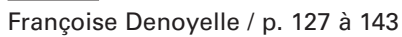


(3) De 1906 à 1938,

Henri Manuel travaille pour trente revues de mode et plus particulièrement pour La femme de France (1922-1935), Les grandes modes de Paris (1906-1931), Les modes de la femme de France (1922-1935), Le petit écho de la mode (1928-1936).

(4) Voir, à ce sujet, Françoise Denoyelle, La lumière de Paris, Les usages de la photographie, (1922-1939), L'Harmattan, Paris, 1997.

(5) Le bilan financier de l'exploitation fait apparaître des pertes pour les exercices 1931, 1934, 1936.

\section{I) Le choix du studio Henri Manuel}

Une commande d'une telle ampleur ne peut être qu'une initiative du ministère de la Justice. Le choix du studio Henri Manuel n'est pas sans surprendre tant sa production semble éloignée de ce genre de reportage. Pourtant, un examen plus approfondi de son histoire justifie la décision du ministère de la Justice. En 1900, Henri Manuel ouvre un studio d'art spécialisé dans le portrait. Très vite il photographie les personnalités du monde de la politique, des arts, du spectacle et des sports. Ses portraits sont peu à peu utilisés par la presse quotidienne et, en 1910, il crée un service de presse chargé de les commercialiser : l'Agence universelle de reportage Henri Manuel, « une très importante entreprise dont le prestige est dû entièrement au talent artistique, à l'esprit d'initiative et aux relations très étendues de Monsieur Henri Manuel dans tous les milieux de Paris ». Loin d'être universelle comme l'indique la publicité, l'agence opère surtout à Paris. En 1925, Henri Manuel loue un immeuble au 27, rue du Faubourg Montmartre. Sur cinq étages, il installe ses ateliers, ses laboratoires et ses services commerciaux. Pour rentabiliser son affaire il diversifie sa production, travaille pour la mode ${ }^{(3)}$ et photographie les robes et les ensembles de Chanel, Lanvin, Patou, Poiret, Schiaparelli... Pendant l'entre-deux-guerres, il est le photographe le plus prolifique dans le secteur de la mode. Près de 100.000 clichés de mode seront vendus au poids du verre pour dégager de la place et rentabiliser la production. Dans les années trente, comme nombre de ses confrères, il s'ouvre à la photographie publicitaire et poursuit ses reportages. À la vente du studio ${ }^{(4)}$ en 1941, l'inventaire fait état d'un fonds comprenant 300.000 clichés de reportages, 350.000 clichés de personnalités et clients récents, 260.000 clichés vendus au poids du verre.

Au début de cette période, le studio est en difficulté. ${ }^{(5)}$ Si le marché est en expansion, la concurrence est importante, particulièrement avec l'arrivée des agences photographiques étrangères. Trouver de nouvelles commandes est impératif. Henri Manuel, habitué à photographier en studio, n'est pas un spécialiste des reportages. Il obtient néanmoins un reportage sur l'hôpital Sainte-Anne en 1930. Ses relations avec les membres du gouvernement et des différents ministères qui viennent se faire portraitu- 
rer dans son studio ont certainement favorisé l'obtention d'une importante commande du ministère de la Justice. Ce genre de reportage "officiel" n'est pas nouveau. Une campagne de prises de vue sur la colonie correctionnelle des Douaires a fait, en 1895, l'objet d'un album de présentation plus élaboré que ceux d'Henri Manuel. Si l'auteur des clichés reste inconnu, un texte introductif présente l'institution qui reçoit des mineurs de treize à dix-sept ans confiés à l'Administration pénitentiaire par les tribunaux, ainsi que des pupilles. Un enseignement primaire ainsi qu'une éducation morale sont assurés par des institutrices. Les pupilles sont initiés aux métiers de l'agriculture et de l'industrie. Peu d'images montrent la vie quotidienne à l'école. Pour l'essentiel, le photographe a opéré comme le font ses confrères dans les pays exotiques. Des scènes de genre présentent, dans un studio improvisé et derrière un fond neutre, les Ouvriers industriels, Un jardinier, Costume du dimanche et de travail, Personnel de surveillance, Pupilles à l'arrivée. Les portraits, très statiques, prennent parfois des allures anthropométriques : Les forts, Les rachitiques, Les petits...

Les reportages d'Henri Manuel répondent à de tout autres ambitions. Plus dynamiques, ils témoignent largement des conditions de vie dans les différents types d'institutions. Faute d'archives, il est impossible de savoir quel était le cahier des charges afférent à la commande, quels étaient les destinataires de ces photographies? Les albums, de facture artisanale, laissent à penser que la diffusion en fut restreinte. L'édition de cartes postales, réservée aux institutions accueillant des mineurs, a-telle été prévue initialement ou décidée une fois la commande réalisée ? Nous ne pouvons apporter aucune réponse à ce sujet. Il nous reste donc à essayer de déterminer, par l'analyse des images, l'esprit qui a présidé au reportage. Nous ne retiendrons ici que les photographies consacrées aux centres pour mineurs, mais les mêmes remarques peuvent s'appliquer aux documents sur les prisons pour adultes.

\section{II) Un reportage exhaustif}

L'ensemble du reportage couvre donc neufs institutions. Cinq d'entre elles sont des centres d'accueil pour les garçons : maisons d'éducation surveillée d'Aniane, de Saint-Maurice, de Belle-Île-en-Mer, école de réforme 
Fig. 1

Le directeur de l'école de préservation de Clermont-de-l'Oise dans son bureau.

Cliché Henri Manuel, collection CNFE-PJJ. de Saint-Hilaire, colonie correctionnelle d'Eysses. Trois autres s'adressent aux jeunes filles : les écoles de préservation de Cadillac, de Clermont-del'Oise et de Doullens. Enfin, la prison de la Petite Roquette détient des garçons et des jeunes filles mineurs.

Les reportages, d'ampleur variable, s'attachent tous à présenter l'ensemble des acteurs : personnel de direction et d'encadrement, enfants de tous âges, visiteurs. Les bâtiments et leurs dépendances, les sites d'initiation à l'agriculture et à la pêche sont, dans la plupart des cas, montrés dans leur fonctionnement.

\section{III) Le personnel d'encadrement}

Le directeur trône invariablement à son bureau. La pose comme les lieux sont austères et incarnent l'autorité. Celui de l'école de préservation de Clermont-de-l'Oise pose la main sur une liasse de documents et, au premier plan, des ouvrages empilés de façon désordonnée se veulent les témoins d'une grande activité. La disposition des dossiers sur un vaste bureau trahit la mise en scène. Le mur du fond est plus révélateur de l'austérité générale des bâtiments. La décoration se réduit à quelques photographies perdues sur un pan de mur d'une pièce haute de plafond.

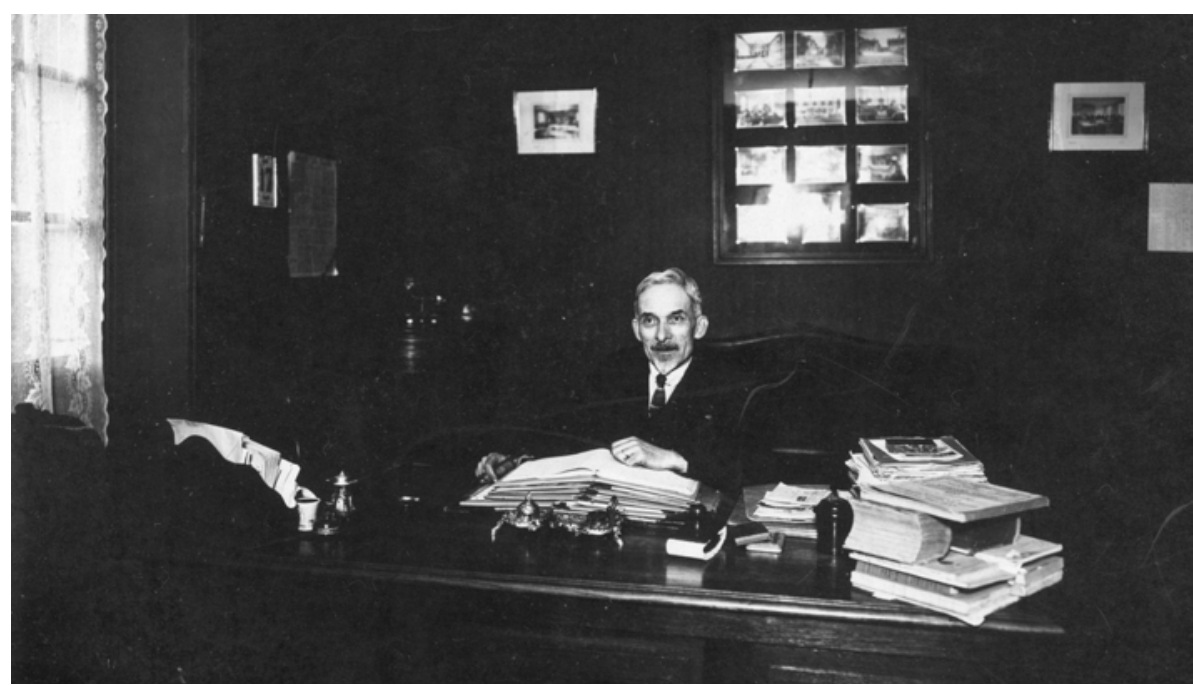


Parfois, la mise en scène va jusqu'à l'organisation d'une situation où les différents protagonistes incarnent, d'une façon symbolique, l'autorité et la soumission. Dans le bureau directorial, est réuni le personnel d'encadrement de la maison d'éducation surveillée de Belle-Île-en-Mer. Le directeur, assisté d'un adjoint, arborant une gravité affectée propre à ne délivrer que des sentences irrévocables, interroge un adolescent en tenue de mousse. En retrait, derrière le bureau, un surveillant, dans une pause un peu gauche, se tient près d'une porte ouverte qui donne sur une seconde salle où trois autres mousses, face contre le mur, attendent visiblement d'être eux aussi interrogés ou punis.

Fig. 2

Audition d'un mineur à la colonie de Belleîle-en-Mer. Cliché Henri Manuel, collection CNFE-PJJ.

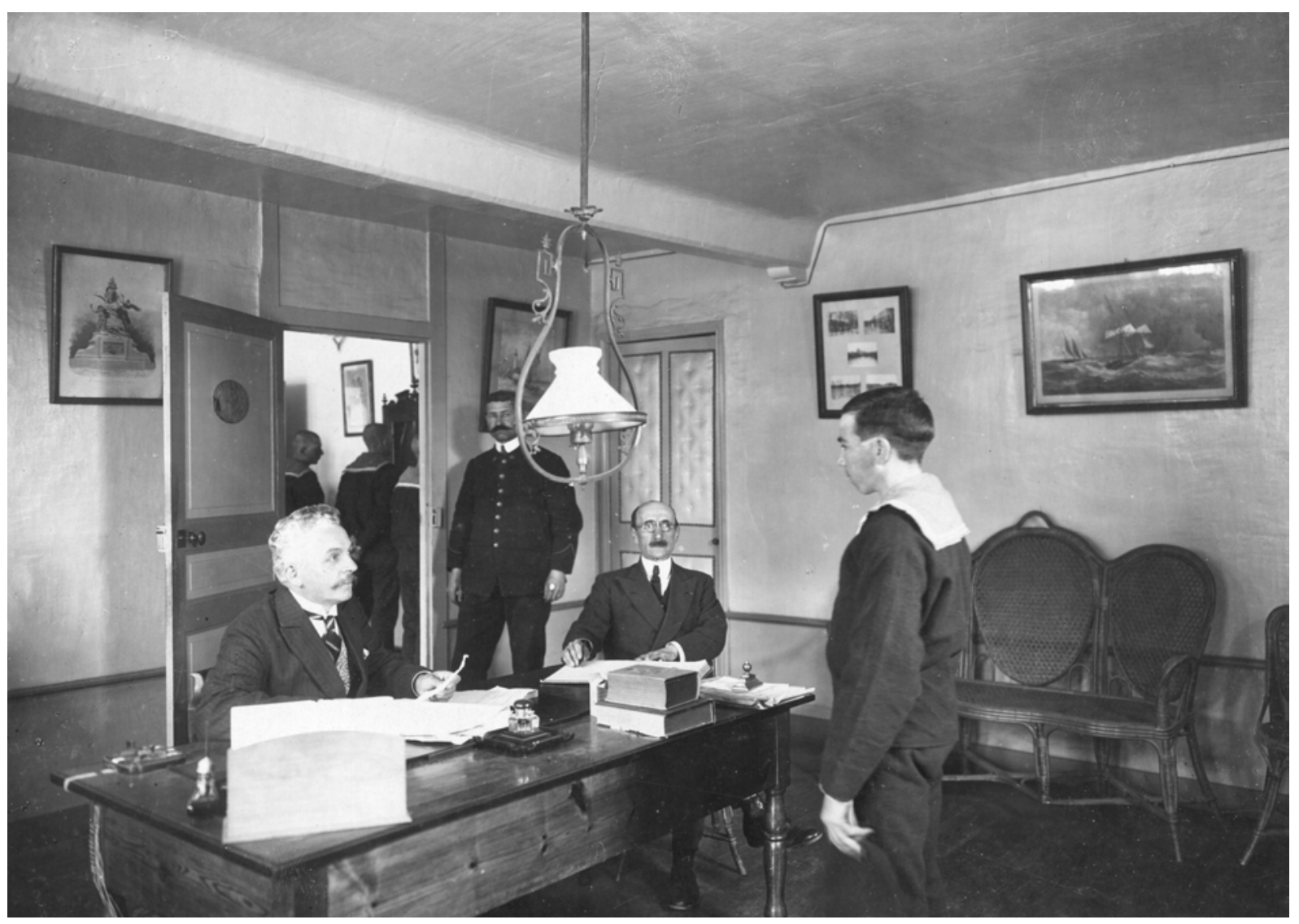


(6) En fait, le règlement des maisons d'éducation surveillée précise que le "prétoire" est dirigé par le directeur de l'établissement et un de ses adjoints.
À la colonie correctionnelle d'Eysses, la mise en scène est moins évidente ; l'incarnation de l'autorité n'en est que plus présente. Une scène de prétoire, "tribunal" disciplinaire des lieux de détention, rassemble deux "juges" en civil ${ }^{(6)}$ assistés d'un surveillant faisant office de greffier. Derrière et en contrebas, un garçon, flanqué d'un surveillant, se tient debout devant ses "juges".

Fig. 3

Le "prétoire" à la colonie

correctionnele d'Eysse.

Cliché Henri Manuel, collection CNFE-PJJ.

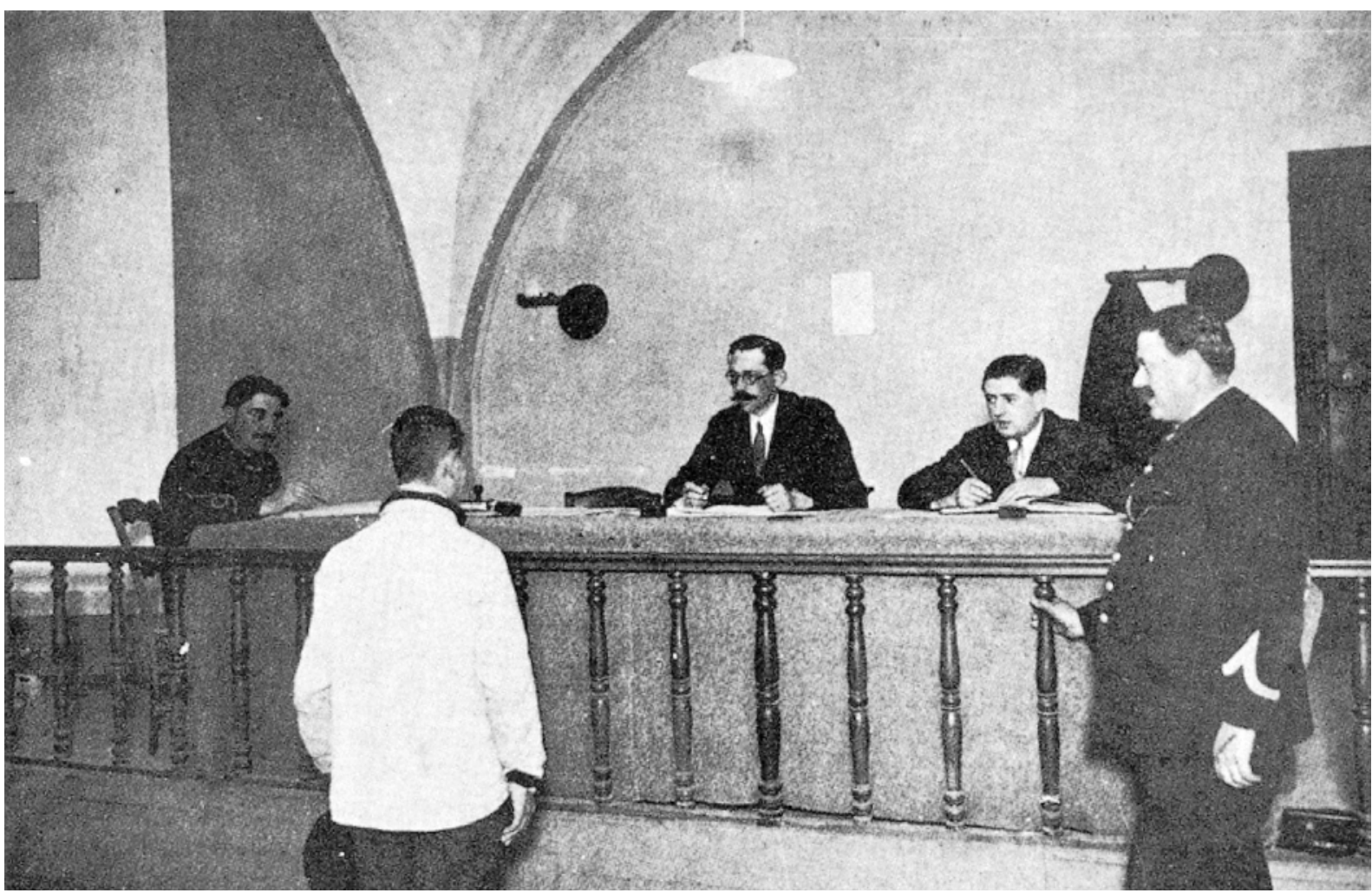


Deux autres photographies de la maison d'éducation surveillée de Belle-Île-en-Mer ont une fonction d'une autre nature. L'une se contente de rassembler les gardiens dans des poses plus décontractées qui laissent à penser que les albums étaient distribués au personnel, l'autre met l'accent sur le rôle pédagogique des adultes. Un repas, sans doute plus copieux qu'à l'ordinaire, réunit des adultes, moniteurs ou enseignants, servis par un mousse. Surveillants, instructeurs et enseignants sont rarement photographiés seuls. Les femmes enseignent aux plus petits et aux jeunes filles. Laïques, elles sont toujours assistées par une fonctionnaire vêtue du strict uniforme de l'Administration pénitentiaire. Les fonctionnaires en uniforme sont également présents au côté des instituteurs des établissements pour garçons.

Fig. 4

La classe à Aniane.

Cliché Henri Manuel, collection CNFE-PJJ.

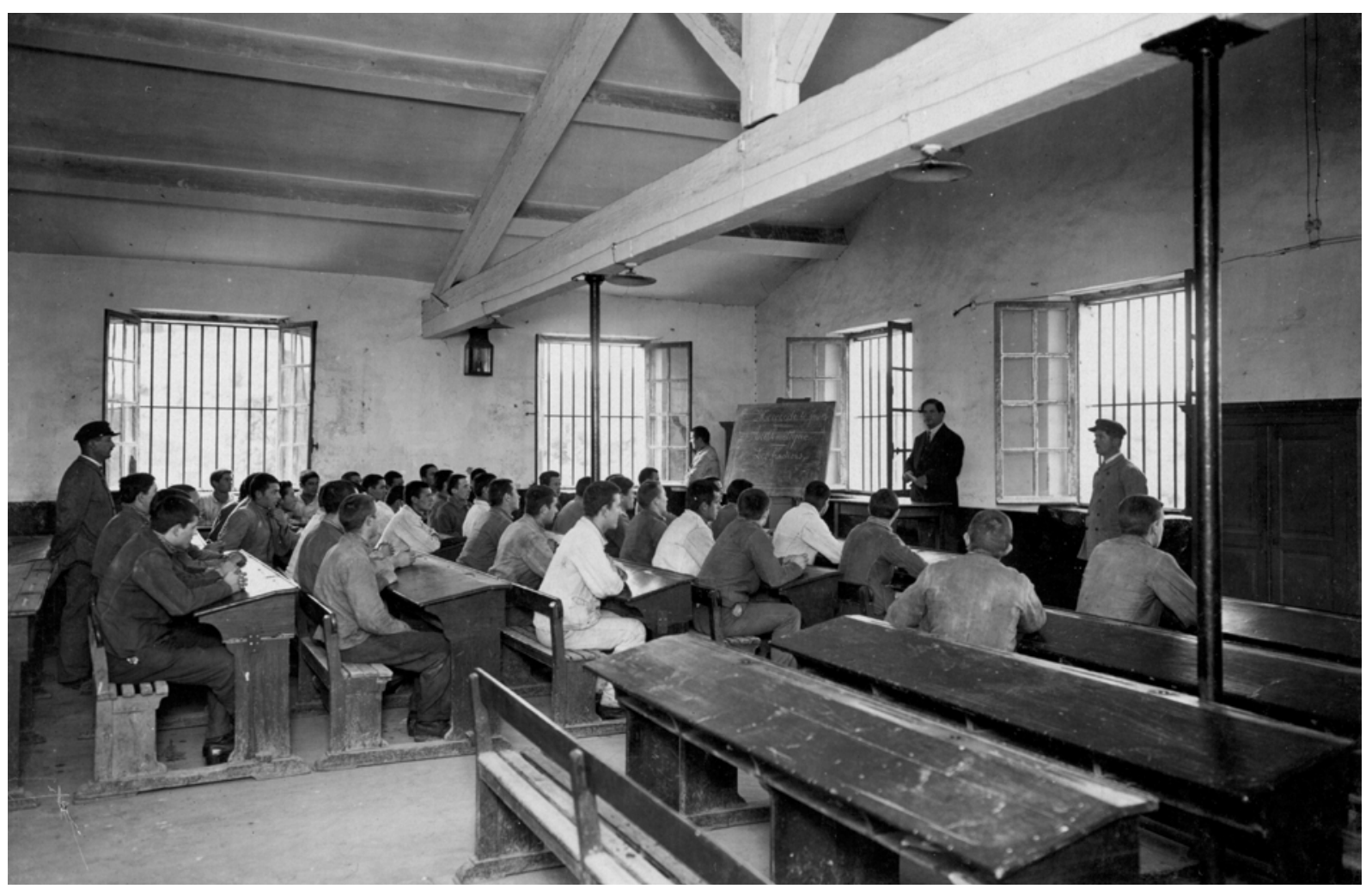


Fig. 5

Visite médicale à

l'école de préservation de Clermontde-l'Oise. Cliché Henri Manuel, collection CNFE-PJJ.
Les instructeurs, affectés aux différents apprentissages, sont photographiés au milieu des jeunes, dans les ateliers ou dans les champs. Le personnel sanitaire n'est présent que dans quelques albums. A l'école de préservation de Clermont-de-l'Oise, une scène présente un docteur, assisté de deux infirmières, interrogeant une jeune fille. Derrière elle, quatre de ses camarades attendent leur tour. Mis à part la table recouverte d'une toile blanche, sur laquelle sont posés quelques flacons et une boîte contenant sans doute seringues ou pansements, et la cuvette pleine d'eau portée par une des deux infirmières, rien ne nous laisse penser que nous sommes dans une infirmerie. La mise en scène a pour objectif de signaler l'existence d'un suivi médical, mais un observateur, même contemporain de l'image, en décrypte facilement le côté artificiel.

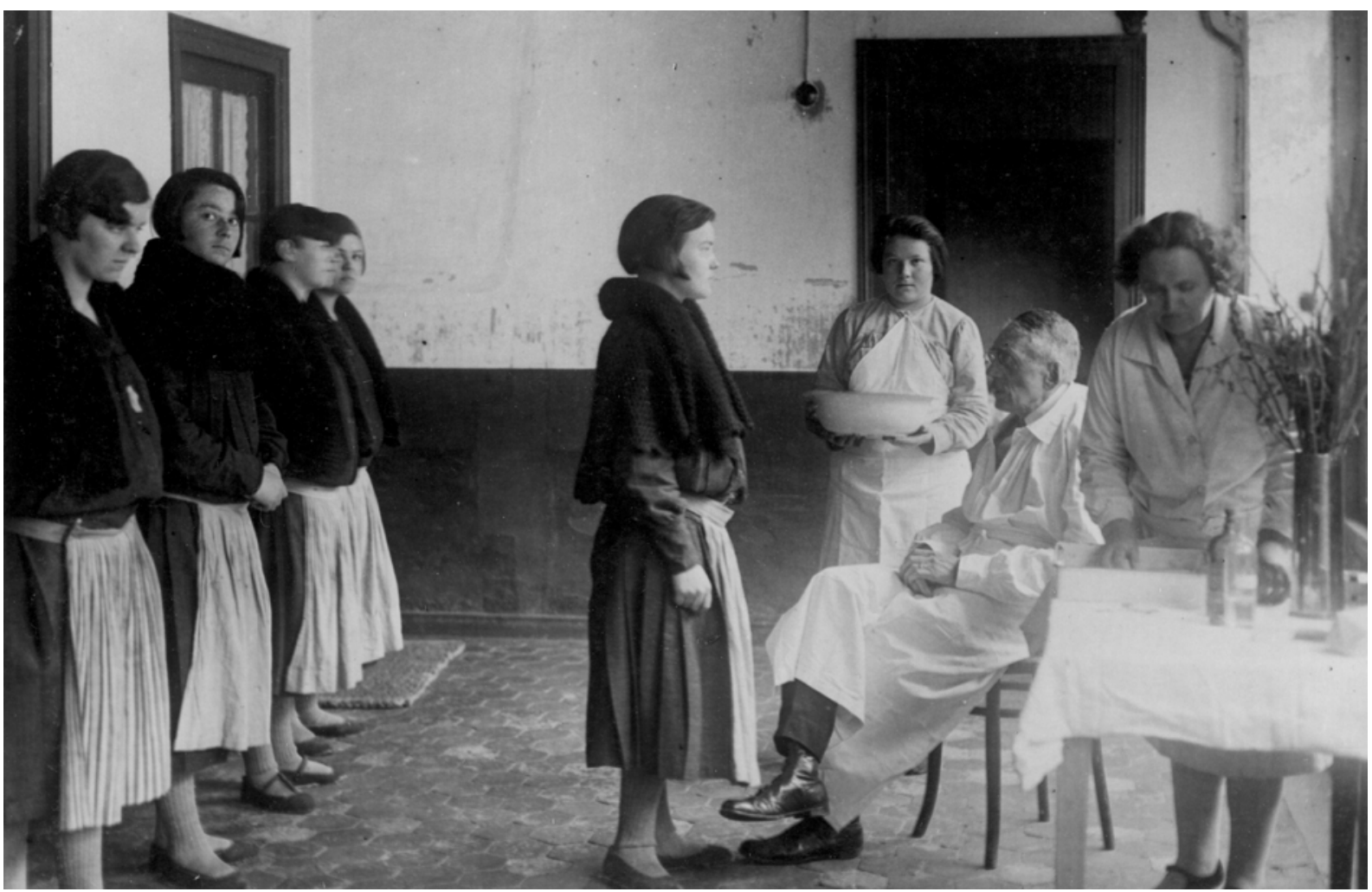


Le personnel, toujours présent sur les photographies, est constamment en situation de travail. Outre ses fonctions de gardiennage, il joue un rôle éducatif, à la fois scolaire et professionnel. L'autorité, bien qu'affirmée, ne prévaut pas sur l'éducation, sauf dans les institutions les plus répressives, comme la prison de la Petite Roquette ou la colonie correctionnelle d'Eysses.

\section{IV) Les enfants et les adolescents}

Garçons et filles, séparés dans diverses institutions, sont le plus souvent appréhendés en groupe lors d'activités : classe, sport, apprentissage... Les adolescentes ont une corpulence révélatrice des régimes alimentaires riches en pain et féculents. Une composition photographique rassemblant les quarts, brocs, écuelles prêts pour le repas souligne la part très importante qu'occupe le pain dans les repas.

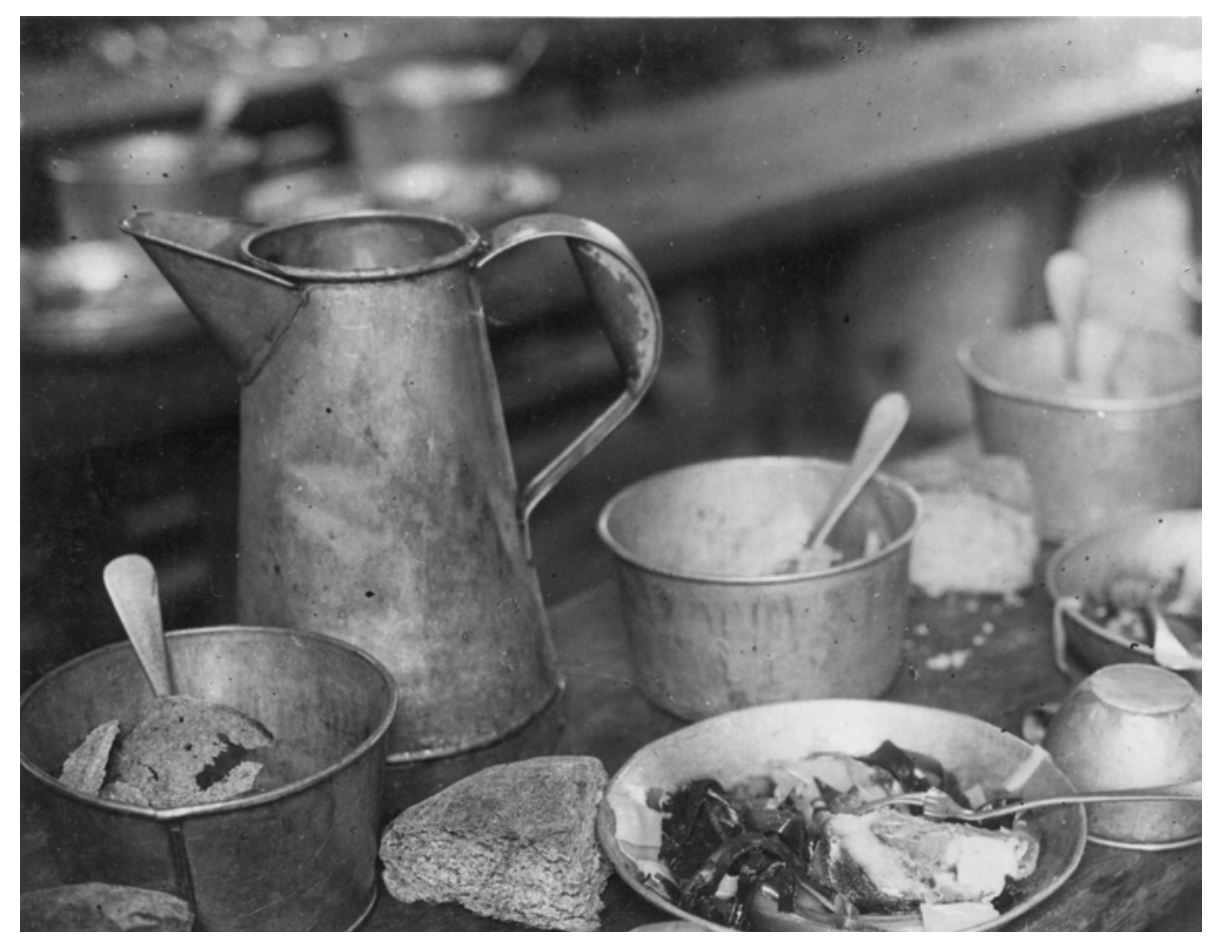

Fig. 6

La "gamelle" à l'école de préservation de Clermont-de-l'Oise. Cliché Henri Manuel, collection CNFE-PJJ. 
Les photographies prises dans les réfectoires lors du déjeuner confirment les informations fournies par la composition. Enfants, adolescents paraissent en bonne santé physique, mais les garçons comme les filles ont rarement le sourire aux lèvres. Ils se contentent de poser comme on leur a demandé de le faire.

Quelques portraits individuels, notamment dans les cellules, sont criants de détresse. Souvent, les adolescents détournent le tête ou se cachent sous une mauvaise couverture posée sur une paillasse. Si certaines prises de vue préservent l'anonymat des enfants et des adolescents, c'est uniquement en fonction d'impératifs photographiques. En classe, ils sont pris de dos afin de présenter de face l'encadrement, les tableaux et le matériel pédagogique accrochés au mur. Dans la boulangerie de l'école de réforme de Saint-Hilaire, les adolescents sont couchés sur le pétrin, mais ceux qui travaillent dans la forge posent de face, tout comme ceux qui chargent des troncs d'arbres sur des charrettes. Dans la basse-cour de l'école de préservation de Doullens, une jeune fille détourne la tête, mais deux autres fixent l'objectif, ce qui laisse à penser qu'aucune instruction précise n'a été donnée à l'opérateur. Les mêmes remarques prévalent pour la colonie correctionnelle d'Eysses et la prison de la Petite Roquette.

La vision que donnent les reportages des enfants et des adolescents est essentiellement collective, inscrite, comme celle du personnel, dans des activités où les deux groupes se côtoient journellement.

\section{V) Les locaux}

Omniprésents comme éléments du décor, les locaux ne peuvent guère être mis en scène et traduisent avec force la réalité quotidienne de l'enfermement, de la vétusté. L'exiguiité côtoie le vaste, tous deux aussi impropres au minimum d'intimité. L'enfermement s'impose dès l'entrée par une vaste porte, des murs d'enceinte élevés, tout comme ceux qui cloisonnent les différents quartiers dévolus à la répression. Le quartier cellulaire de Saint-Hilaire aligne des petites fenêtres protégées par de lourdes grilles et, dans l'étroite cour, les garçons cassent des cailloux à genoux. 


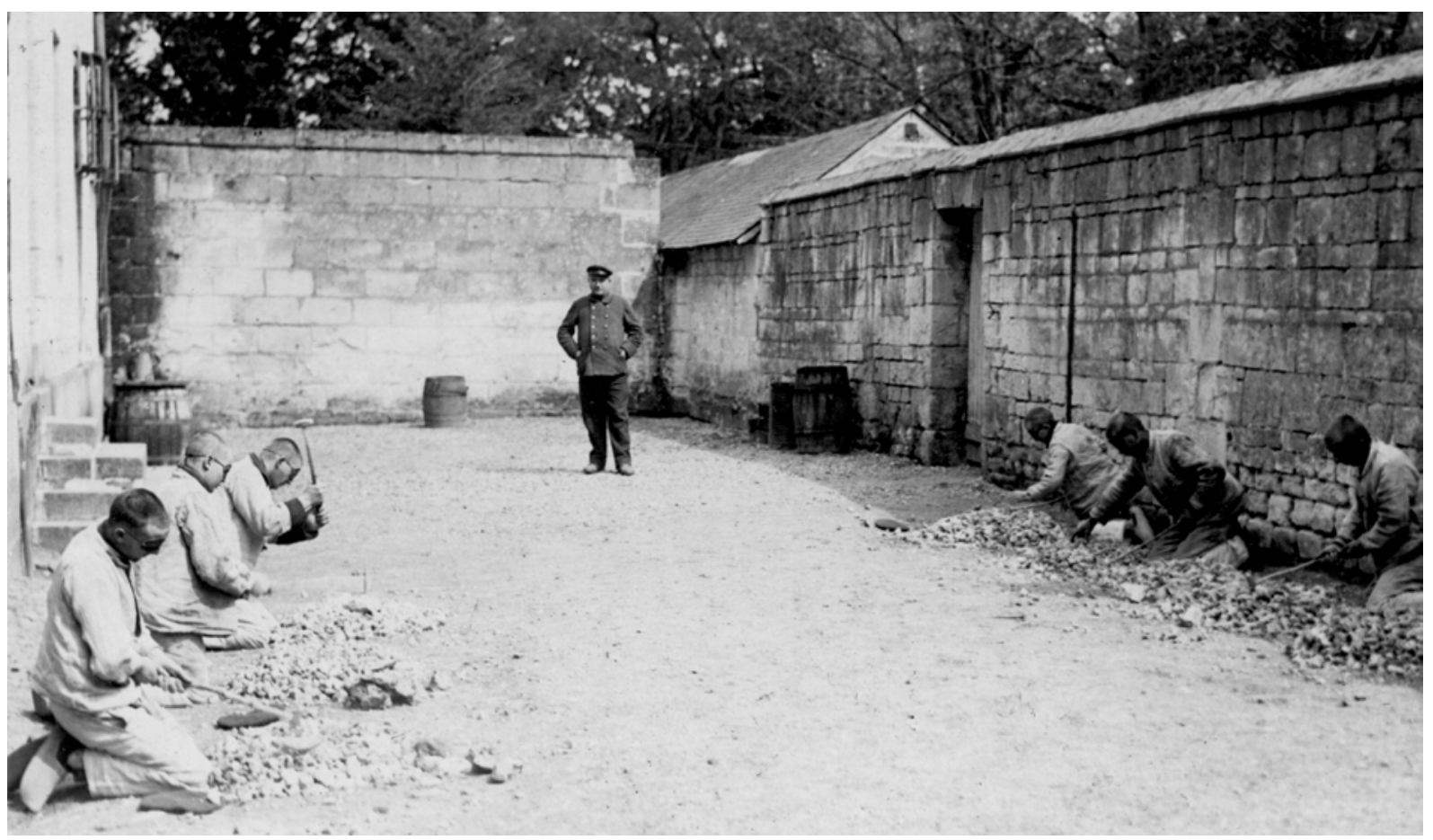

Le décor de la cour de l'école de préservation de Clermont-de-l'Oise est tout aussi austère. Le mur de clôture est encore plus haut et, si deux couples de jeunes filles esquissent un pas de danse, les autres tournent dans la cour sous l'œil vigilant des surveillantes. L'univers carcéral imprime sa marque. À la maison d'éducation surveillée de Saint-Maurice, les activités agricoles se déroulent dans plusieurs bâtiments d'aspect moins austère. Habilement, le photographe a saisi les jeunes jouant au ballon devant un bâtiment plus accueillant : l'infirmerie. Visiblement, ce terrain n'est pas dévolu, habituellement, à ce genre d'exercice.

L'intérieur des bâtiments est appréhendé suivant deux types de fonction : l'hébergement, d'une part, les différentes activités, d'autre part. Les enfants et les adolescents dorment dans des structures allant de la cellule individuelle au dortoir. À l'école de préservation de Doullens, de lourdes portes, avec heurtoir et verrou, disposées le long d'un couloir, ferment
Fig. 7

Quartier cellulaire de Saint-Hilaire. Cliché Henri Manuel, collection CNFE-PJJ. 
chaque cellule. À l'intérieur, l'unique mobilier est constitué d'un lit métallique. Les murs de briques sont passés à la chaux, la fenêtre avec des barreaux donne sur un autre mur. Le sol dallé est en mauvais état. L'univers est carcéral. Dans la même école, une chambre à deux lits séparés par une table de nuit accueille une jeune fille enceinte. Dans l'album, aucune légende n'accompagne la photo, mais la carte postale qui la reproduit indique que nous sommes dans "la salle des expectantes". Le linge est impeccable et on a même disposé une sorte de tapis sur le parquet ciré.

Fig. 8

Carte postale réalisée

à partir de l'album

Manuel sur Doullens.

Collection CNFE-PJJ.

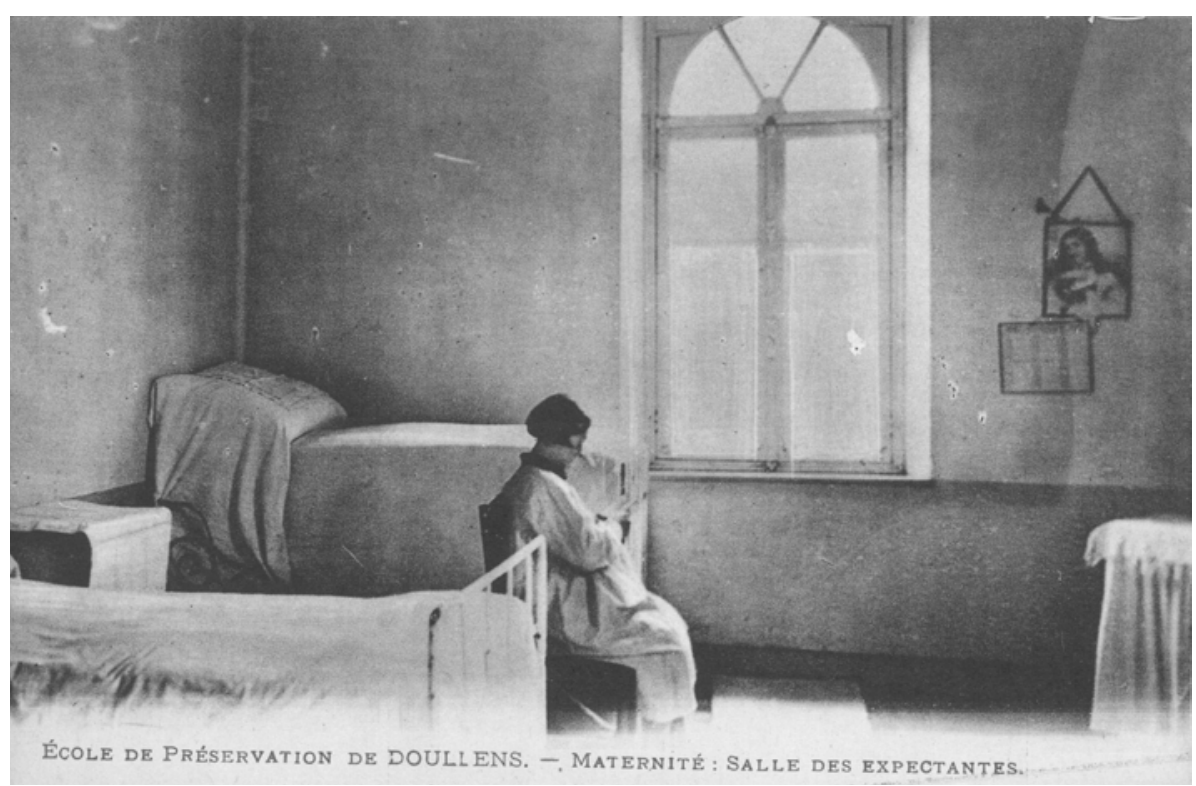


Lorsque les jeunes filles ont eu leur bébé, elles gardent l'enfant avec elles. La chambre accueille alors deux lits. Comme dans la photographie précédente la mise en scène est importante. Draps et serviettes ont été disposés spécialement pour le photographe. En dépit des efforts de l'institution pour donner une image valorisante, le dénuement est total. Les murs sont vides, pas un objet, pas un jouet n'esquisse un semblant de vie dans la pièce. Le dortoir de l'école de préservation de Clermont-de-l'Oise est plus classique et rappelle ceux qui accueillaient les jeunes filles dans les lycées à la même époque. Tout autres sont les cellules de bois, "les cages à poules", aménagées dans une vaste pièce du même établissement. Une vue en plongée traduit l'exiguité de chaque cellule, dont l'espace correspond à celui qu'exige un lit : $1,90 \mathrm{~m}$ x $0,90 \mathrm{~m}$. Les mêmes aménagements se retrouvent dans les établissements pour garçons.

\section{Fig. 9}

"Cages à poules", colonie de

Saint-Maurice.

Cliché Henri Manuel, collection CNFE-PJJ.

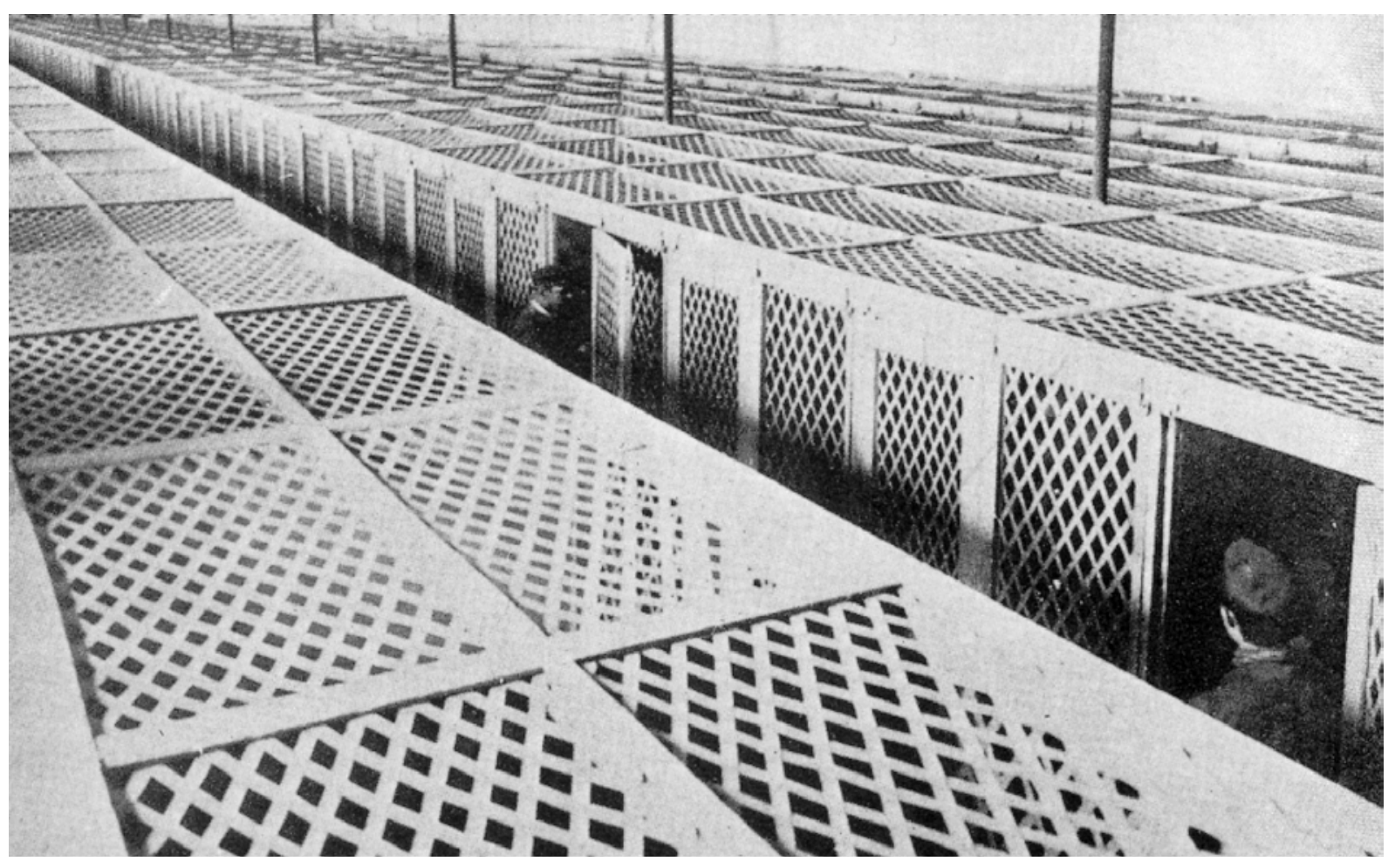


Les repas sont pris dans des réfectoires. Le mobilier est rudimentaire, l'éclairage et le chauffage notoirement insuffisants pour d'aussi vastes salles. À l'école de préservation de Clermont-de-l'Oise, un petit poêle est censé chauffer une pièce de plusieurs centaines de mètres carrés. Ici comme dans les classes, les adolescentes portent des châles sur les épaules et les surveillantes ne quittent pas leur manteau. La vaisselle, entièrement en métal, est usagée. Parfois, la mise en scène a disposé des serviettes en éventail et quelques guirlandes peuvent ajouter un air de fête, mais l'atmosphère est silencieuse. Tout le monde mange, personne ne parle et la surveillante chef, installée sur une estrade, fait régner l'ordre.

L'hygiène, incluse dans la formation des jeunes, donne lieu à quelques vues des installations sanitaires. Lavabos collectifs ou individuels, quelles que soient les institutions, témoignent eux aussi de la vétusté des installations. Les jeunes filles de l'école de préservation de Doullens font semblant de se laver les mains, mais aucune eau ne sort des robinets et le même problème touche les douches. Quant aux baignoires de l'école de réforme de Saint-Hilaire, elles sont probablement réservées aux malades atteints de tuberculose. La vétusté des installations sanitaires ne doit cependant pas occulter un désir de l'administration d'inculquer les règles de l'hygiène, à une époque où l'eau courante est encore absente de bien des logements et où les taudis sont nombreux en ville.

Les locaux consacrés à la formation sont d'aspect variable. Les classes sont les plus accueillantes. Vastes comme toutes les salles consacrées à la vie collective, elles abritent le même modeste poêle, mais le matériel pédagogique - cartes, planches dédiées à la botanique, au système métrique... - est identique à celui utilisé dans les écoles primaires de l'époque. Les images, gravures accrochées aux murs, sont nombreuses. La touche personnelle de l'enseignant est évidente et donne un peu de chaleur à l'ensemble. Les locaux dévolus aux apprentissages n'ont pas toujours été conçus pour cela. Le fournil de l'école de réforme de Saint-Hilaire ne dispose que de fours. Rien n'est prévu pour poser les paniers dans lesquels est disposée la pâte. Les boulangers les ont donc mis par terre. La menuiserie de la maison d'éducation surveillée de Saint-Maurice est une pièce assez petite, si bien que les établis sont trop proches les uns des autres 
pour pouvoir travailler convenablement. En revanche, les matelassières déjà évoquées ont été installées dans une remise où a échoué un confessionnal désaffecté. La pièce est trop vaste pour les six jeunes filles et semble un lieu de travail improvisé pour la photographie.

Peu d'espaces sont dédiés aux distractions, loisirs et à la religion. Généralement, pour chaque institution, une photographie est consacrée à la messe, mais l'esprit laïque interdit toute manifestation ostentatoire de la religion. Les distractions sont rares : chorale à l'école de préservation de Clermont-de-l'Oise, audition d'un disque à la maison d'éducation surveillée d'Aniane, fanfare à la maison d'éducation surveillée de SaintMaurice. Le sport, en revanche, se pratique sous de multiples formes : gymnastique et danse pour les jeunes filles, jeux de ballon, agrès, gymnastique pour les garçons. Il s'agit de développer le corps au même titre que l'esprit, mais aussi de canaliser une énergie. L'enfermement, la garde constante ne laissent guère d'espace de liberté à ces jeunes corps, qui ont vite tendance à s'empâter.

Les locaux constituent un témoignage fort sur les conditions d'hébergement et de vie des enfants et des adolescents. Ils apparaissent comme des lieux froids, lugubres, plus adaptés au gardiennage, à la répression qu’à l'éducation.

\section{VI) Les techniques de reportage}

Le studio Henri Manuel, nous l'avons vu, n'est pas un spécialiste du reportage. Ce handicap se révèle, pour nous qui examinons les photographies soixante-dix ans après leur prise de vue, comme un précieux auxiliaire. Le manque d'habileté des opérateurs ${ }^{(7)}$ met nettement en évidence les mises en scène, comme nous l'avons déjà souligné à plusieurs reprises. Les enfants et les adolescents portent toujours des vêtements qui sortent de la lingerie. Les vêtements pour le travail sont tout aussi propres. Quelques exceptions, comme les pantalons des mitrons de l'école de réforme de Saint-Hilaire, montrent que la réalité quotidienne devait être bien différente. L'aspect vestimentaire des jeunes est du ressort des institutions et traduit leur volonté de se valoriser. Les mises en scène sont plus complexes à analyser. Ont-elles été organisées par le pho-
(7) Compte tenu de l'importance et du nombre de reportages, ceux-ci ont été réalisés par des opérateurs différents. 
tographe ou par la direction ? Probablement par les deux. Il est clair que les jeunes filles de l'École de préservation de Doullens n'ont jamais donné de grain aux poules, du moins dans ces conditions. Leurs gestes sont plus que gauches. Elles imitent la fermière qui prend du grain dans son tablier pour le jeter aux poules, mais leurs blouses propres et fermées par derrière ne sont pas adaptées à ce genre d'activité. De nombreuses scènes sont organisées pour rassembler sur une même photographie les différents moments d'une activité ou d'un apprentissage. Sur une même photographie, les matelassières de l'école de préservation de Cadillac rassemblent la paille, la cardent, cousent les paillasses des cellules. A la maison d'éducation surveillée de Saint-Maurice, échafaudage et échelles ont été posés contre un bâtiment. Sept garçons, sous la direction d'un professionnel, miment les différents travaux de maçonnerie et de couverture. En dehors des mises en scène, les techniques de reportage sont rudimentaires. À une exception près, ni les prises de vue en plongée contreplongée, ni les gros plans ne sont utilisés. Ces techniques, très en vogue dans la photographie de propagande et connues du studio Henri Manuel, ne sont pas reprises. Les vues sont frontales, la lumière naturelle utilisée au maximum. Il semble que les opérateurs n'aient eu d'autres buts que de répondre à la commande. Quelques images prises dans les cellules, témoignage sobre mais implacable de vérité, ne répondent pas à une valorisation de l'institution. Rares, elles s'expliquent mal.

\section{VII) L'esprit qui préside au reportage}

Ce chapitre est celui qui pose le plus de problèmes compte tenu des nombreuses interrogations qui restent sans réponse.

1) Quelles étaient les attentes et les motivations du commanditaire ?

2) Le studio Henri Manuel a-t-il répondu à ces attentes ?

3) A l'époque, comment et par qui les images ont-elles été perçues ?

Néanmoins, apparaît la volonté de montrer que la prison n'est pas seulement un lieu de détention/punition, mais aussi de rachat par le travail. L'éducation, la diversité des apprentissages sont largement développés, valorisés ; en revanche, la répression, les brimades, l'enfermement transparaissent plus qu'ils ne sont affirmés. Si la messe est célébrée, l'ensemble 
du personnel est laïque et sont promues les valeurs de travail et d'obéissance, comme en témoignent les inscriptions dans les salles où se rassemblent les jeunes.

Une vision contemporaine appréhende probablement de façon plus critique l'ensemble des reportages. L'enfermement, le manque d'ouverture sur le monde extérieur, la dureté des conditions de vie ne sont pas les mieux appropriés pour former et préparer ces jeunes à une vie d'adulte. Si la formation professionnelle peut être une voie d'insertion, elle est essentiellement en direction des garçons. Les jeunes filles sont cantonnées à des travaux de couture, comme c'était l'usage à l'époque. Ces reportages, par leur exhaustivité, représentent une commande exceptionnelle de l'État. À la même période, entre 1930 et 1931, François Kollar a réalisé, pour les éditions Horizon de France, un reportage intitulé $\mathrm{La}$ France travaille. Son travail, qui répond aux mêmes impératifs d'exhaustivité que celui d'Henri Manuel, est en revanche d'une qualité artistique nettement supérieure et a fait l'objet d'une publication en quinze fascicules et deux volumes. Les albums d'Henri Manuel, par leur facture artisanale, n'ont pas dépassé le stade d'une diffusion interne à l'institution, même si quelques rares images ont été publiées dans un magazine. 